\title{
Results of the digital co-addition of thirteen Schmidt films of the Virgo cluster of galaxies
}

\author{
A.C. Katsiyannis ${ }^{1}$, S.N. Kemp ${ }^{2}$, D.S. Berry ${ }^{1}$, and J. Meaburn ${ }^{1}$ \\ 1 Department of Physics and Astronomy, University of Manchester, Oxford Rd., Manchester M13 9PL, UK \\ 2 Instituto de Astrofísica de Canarias, C/ Via Lactea, E-38200 La Laguna, Tenerife, Spain
}

Received February 23; accepted April 24, 1998

\begin{abstract}
We have digitally co-added APM scans of 13 Kodak TechPan films of the SE region of the Virgo cluster of galaxies. The $\approx 6.2^{\circ} \times 6.2^{\circ}$ field of the $R$-band films combined with the resolution of $\approx 2$ arcsec pixel ${ }^{-1}$ results in data file sizes of about 222 MBytes. The 13 scanned films have been aligned, co-added, corrected for vignetting effects and cleaned of stellar features. To illustrate the astrophysical uses of this technique, we present high contrast images of a sample of Virgo cluster and background galaxies. Several very faint, but very clearly seen features, such as the interaction between IC 3481, IC 3481A and IC 3483 and filaments outside of the common envelope of NGC 4438 \& NGC 4435, can be seen for the first time. We present an image of the halo of M 87 showing its great extent, and an image of the central regions of the cluster which shows no real evidence for interactions between the galaxies in this region. We also present high-quality images of the previously-identified "jet" and shell features around M 89. We also present an image of the whole field which appears to show large variations in brightness of the intra-cluster medium across the region, with the brightest regions in the northern part in the central regions of the cluster, though we caution against this interpretation until we have investigated the large variation in emulsion sensitivity across individual films more thoroughly.
\end{abstract}

Key words: techniques: image processing — galaxies: structure - galaxies: haloes — galaxies: interactions galaxies: clusters: Virgo cluster

\section{Introduction}

The extensive $\approx 6.2^{\circ} \times 6.2^{\circ}$ observing field of the United Kingdom 1.2-m Schmidt Telescope (UKST) of the AngloAustralian Observatory (AAO), allows the whole (or significant fractions) of nearby clusters of galaxies to be imaged simultaneously. The most sensitive photographic emulsions are required for such observations as most regions of extragalactic objects are of very low surface brightness, and techniques such as photographic amplification or co-addition (Malin 1979) increased the depth to which such faint features of galaxies could be identified on Schmidt plates.

At the end of the 1980s, increased computing capability and disk storage facilities allowed for the possibility of digitally co-adding microdensitometer scans of whole Schmidt plates in order to increase the signal-to-noise ratio $(\mathrm{S} / \mathrm{N})$ and enable the detection of fainter extended features of galaxies, and produce a resultant co-added data array from which fully quantitative results could be obtained. As a preliminary project, Kemp \& Meaburn (1991a) co-added Automatic Plate Measuring (APM) machine scans of 8 IIIaJ sky-limited plates of a field containing the IC 4296 cluster of galaxies, for the purpose of detecting faint haloes around galaxies and other extensive low surface brightness features; the scanned area was about $4.5^{\circ} \times 4.5^{\circ}$. Discoveries were reported in Kemp \& Meaburn (1991a,b, 1993, 1994, 1995) and Kemp (1994) and included: a giant halo of dimensions $\sim 600 \times 200 \mathrm{kpc}$ around a cD galaxy; tidal tails, detached filaments and distortions associated with a pair of interacting galaxies; a warped disk of an edge-on spiral galaxy; a set of five spectacular trails "emanating" from an apparently normallooking lenticular galaxy; a possible "shell" feature associated with a late-type spiral galaxy; faint optical emission spatially associated with the radio lobes of IC 4296; and a halo surrounding an irregular SMC-like galaxy which is almost perfectly circular in projection. The faintest features were estimated to be at approximately $27 \mathrm{mag} \operatorname{arcsec}^{-2}$ in the $B_{\mathrm{J}}$ band, while surface brightness profiles could be followed to about $1 \mathrm{mag} \operatorname{arcsec}^{-2}$ below this. A full photometric calibration of this data is now being carried out using Anglo-Australian Telescope (AAT) and South African Astronomical Observatory charge-coupled-device (CCD) data. 
Recently, the availability of hyper-sensitised Kodak Technical Pan (TechPan) films (Kodak 1981; Russell et al. 1992) at the UKST has increased the possibilities for projects studying faint features of galaxies, at least for the red end of the spectrum. Phillipps \& Parker (1993) carried out tests on two films during the initial trials of TechPan film at the UKST. These used the same OG590 filter and plateholder combination employed for normal "OR" $R$ band IIIaF exposures. The difference in quality between the films and the IIIaF plates was easily noticeable visually, the galaxy images on the TechPan films having much greater clarity, due to the Tech-Pan emulsion having extremely fine grain size, low rms diffuse granularity and high resolving power (Phillipps \& Parker 1993 and references within). In good seeing, sky-limited exposures with TechPan films are reported to go a magnitude or more deeper than those with the equivalent IIIaF plates. They found a limiting isophote for galaxy surface photometry of $\sim 27 \mathrm{mag} \operatorname{arcsec}^{-2}(0.2 \%$ of the sky background) for these films.

The RCA CCD combined with the Anglo-Australian Telescope (3.9 m aperture) would take only 2 minutes to achieve the same $\mathrm{S} / \mathrm{N}$ (i.e. pre unit area for low surface brightness sources) as obtained in the individual films. However, the greater sensitivity of the CCD, for any project which involves observing extended objects or fields, is more than offset by the cumulative exposure involved in mosaicing a large number of fields. Modern CCDs, on $4 \mathrm{~m}$-class telescopes, typically cover fields of the order of 0.01 square degrees compared with 40 square degrees for UKST plates. In addition, the mosaicing of CCD fields creates problems with matching discontinuities between adjacent fields. Of course, the two types of detector are complementary, in that objects identified as interesting in the co-added array can be targeted for multicolour CCD follow-up observations, which can give information on the stellar populations contained in them etc. and we will be reporting such work in the future.

After the success of the initial digital plate co-addition project of Kemp \& Meaburn (1991a), the Virgo cluster became the natural follow-up target, as it is the nearest rich cluster of galaxies and has a high galactic latitude which minimises foreground galactic contamination. Thirteen sky-limited TechPan $R$-band films of the Virgo cluster were used. The $\approx 6.2^{\circ} \times 6.2^{\circ}$ area of each film was digitally scanned and the resultant arrays co-added as described below, to produce a digitally co-added array of unprecedented area. New software had to be developed to cope with the alignment and co-addition of such large data files, and for making a non-symmetric vignetting correction to the stacked image. With the co-addition of 13 films galaxy surface photometry is expected to be possible to below $28 R \mathrm{mag} \operatorname{arcsec}^{-2}$ ( $0.1 \%$ of sky) over large angular scales. Initial priorities for investigation included: study of warping in the 20 or so edge-on disk galaxies contained in the area; giant haloes around elliptical galaxies, including M 87; comparison of the low surface brightness intra-cluster medium with maps of the cluster at other wavelengths e.g. X-ray and radio; and a general study of the amount of baryonic dark matter which is visible at very low surface brightnesses. In this paper we assume all Virgo cluster members are at a distance of $17 \mathrm{Mpc}$ (Mould et al. 1995), resulting in a spatial scale of $5 \mathrm{kpc} \operatorname{arcmin}^{-1}$.

This paper reports the software developed and utilised to produce the co-added array of unprecedented spatial area. Some preliminary results are then displayed and described for a number of galaxies in the field. In some cases e.g. M 87 and M 89 well-known galaxies and previously discovered features are seen with the new clarity of TechPan film and new depth produced by the co-addition. In other cases e.g. NGC 4435/4438 and IC 3481/3481A new low surface brightness features indicating interactions between galaxies or disturbances in the outer parts of galaxies have been discovered. Further papers will report these and follow-up CCD observations in more detail.

\section{Observations and data reduction}

The thirteen films of the South-East area of the Virgo cluster of galaxies were taken with the UKST between 1991 and 1994 using the Kodak TechPan emulsion with the OG590 filter. The filter has a lower wavelength cutoff around $590 \mathrm{~nm}(10 \%$ transmittance at $580 \mathrm{~nm}$ and $90 \%$ at $600 \mathrm{~nm}$ ), while the emulsion has an upper wavelength cut-off around $690 \mathrm{~nm}$. This particular combination of filter and emulsion has a very small colour term compared with standard $R$ band (Phillipps \& Parker 1993). The field of the plates covers an area of $6.2^{\circ} \times 6.2^{\circ}$, affected by vignetting. The films used, dates and exposure times, are given in Table 1. The first eight films were from the UKSTU archive, while the remaining five were taken specifically for this project. A sixth film (OR16137) was also taken for this project, but has double images and so was not used.

The $\approx 6.2^{\circ} \times 6.2^{\circ}$ area of the films were scanned using the APM machine at the Royal Greenwich Observatory, Cambridge, at a pixel size of $32 \mu \mathrm{m}\left(\approx 2^{\prime \prime}\right)$. The size of the digitized files was approximately $10500 \times 10500$ pixels, which occupied about 222 MBytes.

The thirteen raw frames were combined into a single co-added image using applications from the Starlink KAPPA and CCDPACK packages (see Starlink User Notes 95 and 139, available on the World-WideWeb through the Starlink home page at http://starWWW.rl.ac.uk/). Some of these applications were modified to handle the very large image size by breaking the input image up into "chunks" of $512 \times 512$ pixels for processing. The facilities of the NDF subroutine library were used to do this (see Starlink User Note 33). The following steps were involved in creating the final co-added image: 

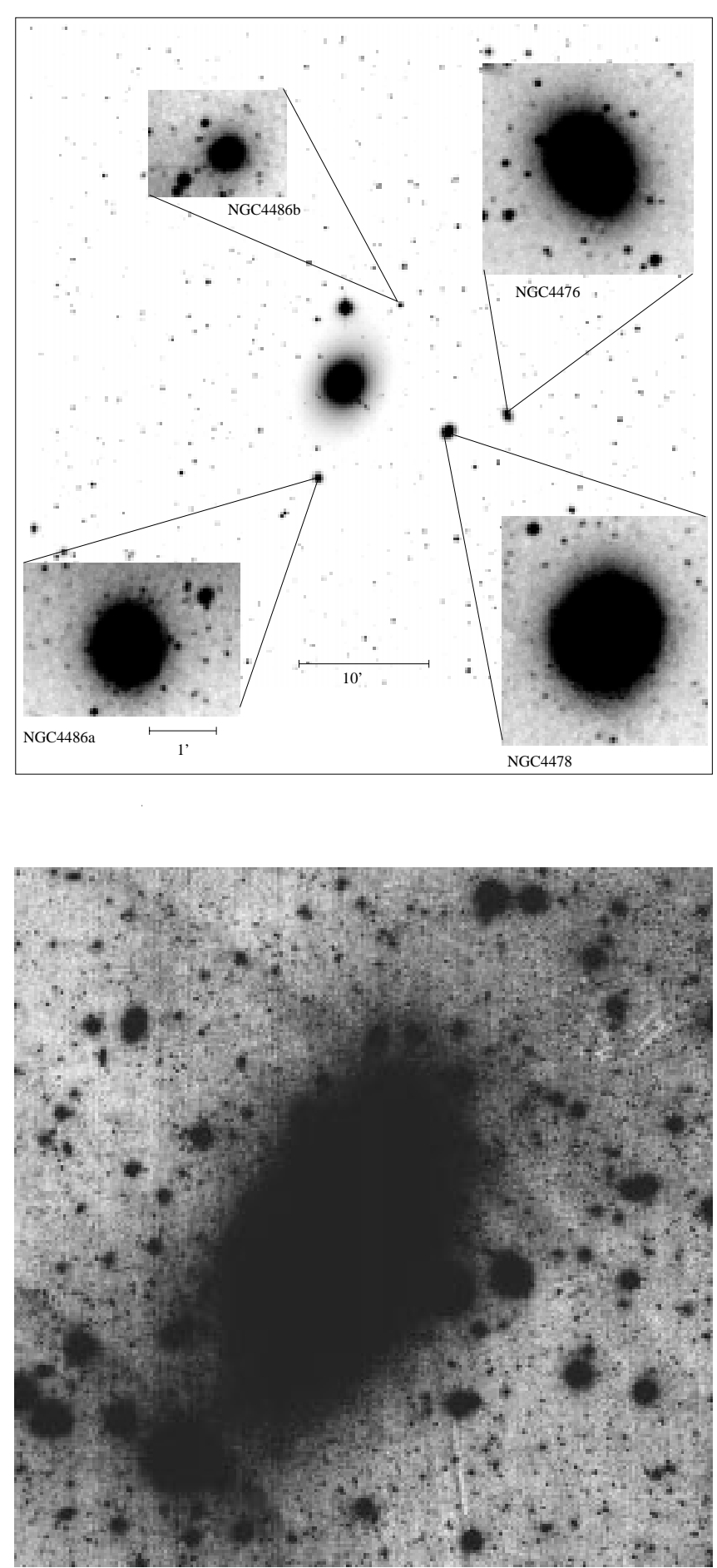

Fig. 1. a) Normal contrast images of $\mathrm{M} 87$ and high contrast images of its companions NGC 4476, NGC 4478, NGC 4486b and NGC 4486a. All the small images are the same scale. The $1^{\prime}$ scale is for the inset images and the $10^{\prime}$ for the main image. b) A deep, high contrast images of $\mathrm{M} 87$, covering the same area as the normal contrast image in Fig. 1a. The dimensions of the frame are $\sim 50^{\prime} \times 50^{\prime}$
Table 1. Details of the films

\begin{tabular}{||c|c|c|}
\hline Name of Film & Exposure & Date \\
\hline- & $(\min )$ & $(\min )$ \\
\hline OR14310 & 75 & $16 / 4 / 1991$ \\
OR14322 & 100 & $20 / 4 / 1991$ \\
OR14782 & 60 & $28 / 2 / 1992$ \\
OR14787 & 60 & $29 / 2 / 1992$ \\
OR14805 & 60 & $9 / 3 / 1992$ \\
OR14811 & 60 & $10 / 3 / 1992$ \\
OR14820 & 70 & $12 / 3 / 1992$ \\
OR14831 & 70 & $14 / 3 / 1992$ \\
OR15980 & 65 & $11 / 3 / 1994$ \\
OR15985 & 70 & $12 / 3 / 1994$ \\
OR15996 & 60 & $19 / 3 / 1994$ \\
OR16073 & 60 & $11 / 5 / 1994$ \\
OR16167 & 60 & $1 / 7 / 1994$ \\
\hline
\end{tabular}

- Conversion of the tape FITS files to Starlink NDF format.

- Alignment of the thirteen frames. For each frame, the centres of a common set of twelve stars located towards the corners of the images were found using a centroiding technique. Transformations were then found which mapped the star centres from each frame onto the corresponding star centres in the first frame. These transformations were of the form:

$x_{\mathrm{out}}=C_{1}+C_{2} \cdot x_{\mathrm{in}}+C_{3} \cdot y_{\mathrm{in}}$

$y_{\text {out }}=C_{4}+C_{5} \cdot x_{\text {in }}+C_{6} \cdot y_{\text {in }}$

where the coefficients $C_{1}$ to $C_{6}$ were chosen to minimise the sum of the squared residuals between the transformed star positions $\left(x_{\text {out }}, y_{\text {out }}\right)$ and the corresponding star positions in the first frame. The typical values found for the coefficient values and the values corresponding to already aligned frames can be found in Table 2. A simple linear transformation was used even though the images cover a large area of the sky. This was possible because the original images were mis-aligned by a very small amount.

The maximum shift obtained from these values does not exceed fifteen pixels for a frame of $10500 \times 10500$ pixels. The accuracy of the alignment is less than a quarter of a pixel.

- Photometric normalisation. The aligned frames were then compressed by replacing each box of $21 \times 21$ pixels by the mean of the pixel values within the box. An exposure factor and zero point offset were then found for each of the compressed images using the makemos application from the CCDPACK package. The full sized images were then modified using the zero point offset and exposure factor for the corresponding compressed image by subtraction of the zero point offset, and division by the exposure factor. This results in the images having a common mean photometric calibration.

- Co-addition. The thirteen frames were then combined into a single stacked image by finding the median of 
Table 2. List of coefficient factors

\begin{tabular}{||c|c|c||}
\hline $\begin{array}{c}\text { Name of the coefficient } \\
\text { factors }\end{array}$ & $\begin{array}{c}\text { Typical values obtained } \\
\text { when aligning the images }\end{array}$ & $\begin{array}{c}\text { The values corresponding } \\
\text { to already aligned images }\end{array}$ \\
\hline$C_{1}$ & -0.7 & 0 \\
$C_{2}$ & 0.9997 & 1 \\
$C_{3}$ & -0.001 & 0 \\
$C_{4}$ & -0.7 & 0 \\
$C_{5}$ & 0.0008 & 0 \\
$C_{6}$ & 0.9997 & 1 \\
\hline
\end{tabular}

the thirteen corresponding input pixel values at each output pixel. The median was chosen rather than the mean in order to eliminate, as far as possible, artifacts such as satellite tracks and scratches which do not occur at the same places in all thirteen images. RA and Dec. information were also stored in the final co-added array.

- Correction for the effects of vignetting. A procedure was developed to reduce the effects of the vignetting caused by the telescope in the periphery of the stacked image. The stacked image was first compressed by a factor of 21 in order to speed up the calculation of the correction. All features significantly brighter than the local background level were then removed, and the resulting image partitioned into two images; one containing all the areas judged to be unaffected by vignetting, and another containing all the vignetted areas. Smooth surfaces were then fitted to each of these two images, and used to estimate the background levels at every point in the vignetted regions. Thus the first fitted image contains an estimate of the unvignetted background level extrapolated from the unvignetted regions, and the second fitted image contains an estimate of the vignetted background level. The ratio of these two images was stored as a correction factor image and expanded to the full image size using bi-linear interpolation. The vignetted areas of the stacked image were then multiplied by this correction factor image, leaving the unvignetted areas unchanged.

- Removal of stellar features. When the stacked image is compressed, the stars in each $21 \times 21$ pixel bin are combined together and result in an extended surface brightness in the compressed image which depends on the number of stars in each bin. To remove this artificial surface brightness, a procedure was developed which automatically removed all stellar-sized features from the full-sized stacked image, prior to compression. This procedure band-pass filters the image to attenuate heavily all features except those of stellar size. Bright features in this image were identified and the corresponding pixels in the original image were removed by setting them to a value which indicates that the the pixels should be ignored. This basic procedure was refined to retain foreground stars which are co- incident with extended background features such as galaxies.

- Gaussian filtering. The data area containing the Virgo galaxy M 89 was smoothed by a two-dimensional Gaussian function with a full-width at half-maximum (FWHM) box of $40 \times 40$ pixels. The resulting image was subtracted from the original data, so any "sharp" features (like shells, jets, filaments, etc) can now be clearly seen.

Multiple $R$-band CCD exposures of a $\approx 6^{\prime} \times 6^{\prime}$ field containing the IC 3481 and IC $3481 \mathrm{~A}$ galaxies were taken in photometric conditions on 1995 Dec. 22-23, using the CCD camera with Tektronix CCD on the $1.0 \mathrm{~m}$ Jacobus Kapteyn Telescope (JKT), La Palma. The total exposure time was $70 \mathrm{~min}$ and the resultant co-added exposure is about as deep as the co-added array in terms of surface brightness. Standard applications from the Starlink KAPPA and CCDPACK packages were used for the reduction of this data and the resulting image (along with others) will be used for calibrating the co-added UKST data. Preliminary results from this calibration are presented below.

\section{Results}

We present five areas of this data array, showing large faint extensions of several galaxies, revealing interactions, common envelopes and shells.

\subsection{The giant elliptical galaxy $M 8^{r} 7$ and its vast extended halo}

M 87 (NGC 4486) is the largest, most massive galaxy in the Virgo cluster. Its radial velocity is $1282 \pm 9 \mathrm{~km} \mathrm{~s}^{-1}$ (de Vaucouleurs et al. 1991) and it is likely to be the central object of the cluster. Arp \& Bertola (1969) suggested a diameter of almost $1^{\circ}$ at the $27 B$ mag $\operatorname{arcsec}^{-2}$ isophote level, also an image of its faint envelope has been presented by Kormendy \& Bahcall (1974). Weil et al. (1997) presented a $B$-band image of M 87 by photographically co-adding five IIIa-J UK Schmidt plates. An asymmetry was seen in the form of a diffuse "fan" of stellar material extending out to about $100 \mathrm{kpc}$ to the SE along the 
projected major axis. They show that accretion of a small spheroidal galaxy into a larger potential can account for this structure.

A normal contrast image of M 87 from the co-added data array is shown in Fig. 1a, in which four neighbouring galaxies are indicated and enlarged at higher contrast. A high contrast image of the same field is also presented in Fig. 1b. The approximate dimensions of the halo in this field are $49^{\prime} \times 25^{\prime},\left(\sim 250 \times 125 \mathrm{kpc}^{2}\right)$, to a surface brightness of approximately $28 R$ mag $\operatorname{arcsec}^{-2}$, which is large, but not as large as some cD haloes (e.g. A3571, Kemp \& Meaburn 1991a, which is of the order of $600 \times$ $200 \mathrm{kpc}^{2}$ ), although it shows the same increase of ellipticity with radius as most $\mathrm{cD}$ haloes. The asymmetry of M 87 noticed by Weil et al. is independently confirmed by our image, although it is not as prominent as it is on the blue plates.

We note that the X-ray profile of this galaxy is much more extensive, Fabricant \& Gorenstein (1983) show it reaching a radius of at least $100^{\prime}(500 \mathrm{kpc})$. The mass of material contained within this radius is of the order of $10^{13} M_{\odot}$ (Fabricant \& Gorenstein 1983; Nulsen \& Böhringer 1995). Whether or not there is a direct relationship between the material detected optically and that observed at X-ray wavelengths is unclear, as is whether the optical material also continues outwards to radii of $500 \mathrm{kpc}$ or greater at lower surface brightnesses. Note that the curved "filaments" on either side of M 87 in the high contrast images are circular "defects" (caused by reflection of the plate holder, S. Tritton, private communication) on one of the original films, not quite removed by the median-filtering addition.

The dwarf ellipticals NGC 4476, 4478, 4486a and b are all optically close to M 87 in projection (see Fig. 1a). In the present data, there is no obvious evidence for interaction between M 87 and any of these galaxies, as there are no features indicating such interaction at the outer isophote levels of these galaxies (distortions, elongations, filaments etc.). The redshifts of NGC 4476, 4478 and 4486b all indicate that they are likely members of the Virgo cluster, though NGC 4476 would have a high relative velocity of $\sim+500 \mathrm{~km} \mathrm{~s}^{-1}$ compared with M 87. NGC 4486a appears to be a foreground object at $\sim+270 \mathrm{~km} \mathrm{~s}^{-1}$. NGC $4486 \mathrm{~b}$ has been classified as a prototype compact elliptical (Rood 1965) though the results of Prugniel et al. (1987) suggest that it could equally be classified as a normal elliptical, but they find NGC 4478 to have the truncated profile of a compact elliptical.

\subsection{The centre of the cluster}

We present normal and high contrast images of the central region of the Virgo cluster, covering a field of $43^{\prime} \times 38^{\prime}$ (see Figs. 2a and b). The giant elliptical galaxies M 84 and M 86 are very prominent in this image, and there are several almost edge-on disk galaxies (NGC 4388, NGC 4402 and NGC 4425) and dwarf elliptical galaxies (NGC 4387 and NGC 4413). This field is in the vignetted region of the films and there is a noticeable variation in the background across this field at high contrast. Linear marks, which can be scratches on an individual film or satellite tracks, can be seen in the field, and there are several blemishes ("white marks") in the SE region. Each of these defects only occurs in one of the set of 13 films, but the variation in backgrounds over the set of 13 films (including the relative variations across films caused by the variations in emulsion sensitivity, see Sect. 3.6) results in median-filtering not being able to remove such features completely, and this is especially noticeable at high contrast in the vignetted areas.

M 86 (NGC 4406) is a giant elliptical galaxy $\left(\mathrm{SO}_{1}(3)\right.$ E3) with a high negative redshift, $-227 \mathrm{~km} \mathrm{~s}^{-1}$ (Binggeli et al. 1985) compared to the average velocity of the cluster $\left(\sim 1150 \mathrm{~km} \mathrm{~s}^{-1}\right.$; Faber et al. 1989) and its velocity dispersion (762 $\mathrm{km} \mathrm{s}^{-1}$, Rangarajan et al. 1995). Forman et al. (1979) suggest that the galaxy is on a radial orbit, passing through the centre of the cluster about every $510^{9}$ years. It is also a luminous $\mathrm{X}$-ray object $\left(L_{\mathrm{X}} \sim 1.7\right.$ $10^{42} \mathrm{erg} \mathrm{s}^{-1}$ ) with most of the X-rays radiated by thermal bremsstrahlung from the interstellar medium, and has a well-studied plume of stripped material to the NW (Rangarajan et al. 1995), reaching 12' (60 kpc) from the centre. The dimensions reached by the halo of M 86 in Fig. 2 are $24^{\prime} \times 16^{\prime}\left(120 \times 80 \mathrm{kpc}^{2}\right)$ which is comparable to the dimensions of the X-ray halo. Rangarajan et al. (1995) and others have suggested that the intra-cluster medium of Virgo is ram-pressure stripping the ISM of M 86, producing the plume and other features visible at X-ray wavelengths. Nulsen \& Carter (1987) reproduce a deep image of M 86 obtained by Malin, showing distortions in its outer isophotes due to regions of excess optical emission associated with features in the X-ray emission, and they suggest that stars are forming from the cooling hot gas in these regions, though Rangarajan et al. (1995) suggest that the excess optical emission is due to scattering of starlight from a high dust concentration.

M 84 (NGC 4374) is a giant elliptical galaxy (E1) with an extended halo of $21^{\prime} \times 18^{\prime}\left(105 \times 90 \mathrm{kpc}^{2}\right)$. It has an total X-ray luminosity of $1.410^{41} \mathrm{erg} \mathrm{s}^{-1}$ detected by Fabbiano et al. (1992) within $1.5^{\prime}(7.5 \mathrm{kpc})$ of its centre (i.e. the optical emission is considerably more extended than the $\mathrm{X}$-ray emission). The galaxy appears to be a weak radio source (3C 272.1; Laing et al. 1983). An organised pattern of Faraday rotation is detected by Laing \& Bridle (1987) at 1.4 and $4.6 \mathrm{GHz}$, implying the existence of a magnetoionic medium in front of the radio-emitting plasma - Laing $\&$ Bridle also suggest that this medium may be responsible for the diffuse component of M 84's X-ray emission.

Despite their apparent proximity, our data offers no evidence for an interaction between M 84 and M 86 . Although the outer isophotes as seen in Fig. 2b almost 

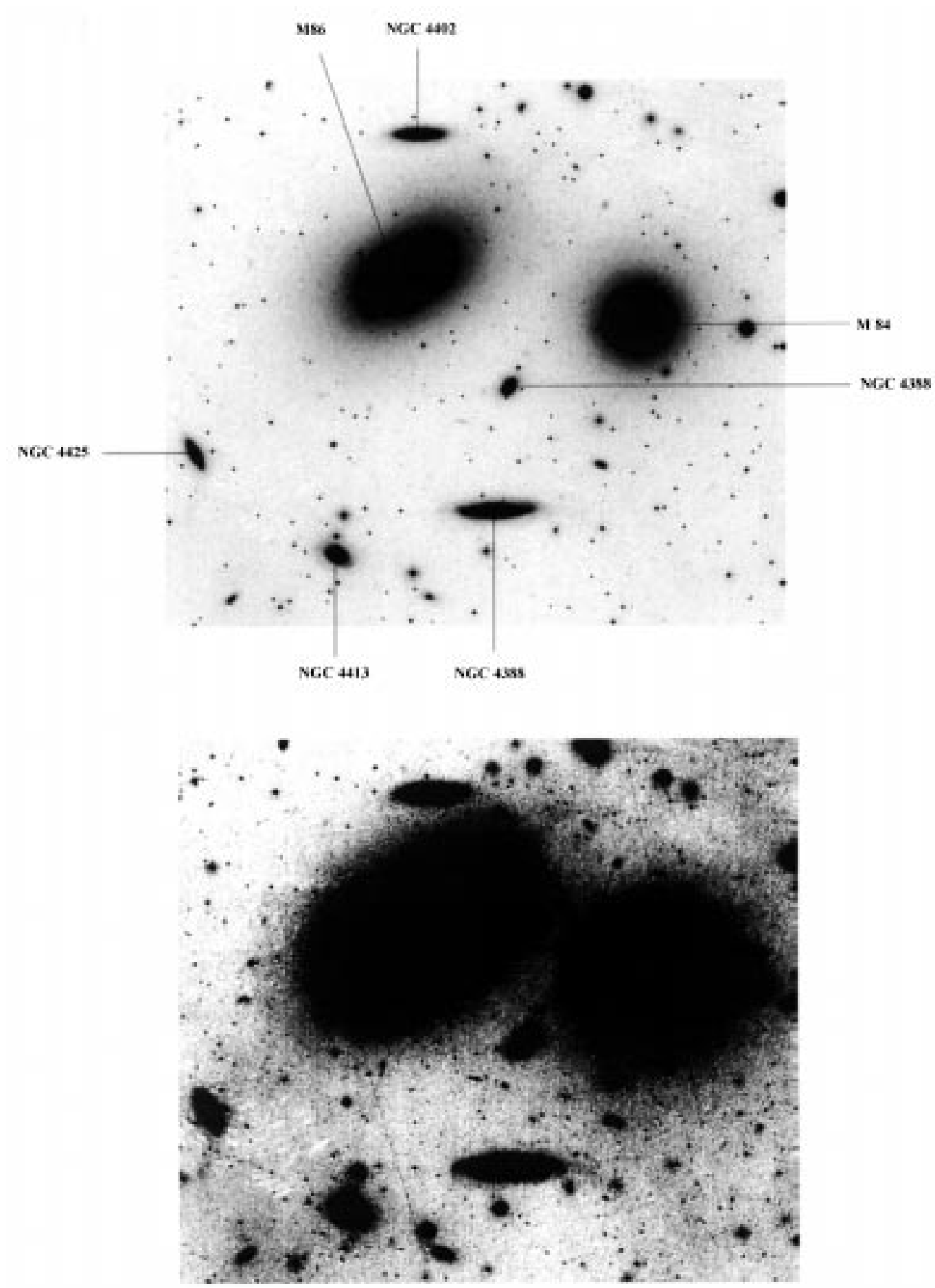

Fig. 2. Normal a) high contrast b) images of the centre of the Virgo cluster of galaxies containing the galaxies $\mathrm{M} 84$, M 86, NGC 4387, NGC 4388, NGC 4402, NGC 4413, NGC 4425. The dimensions of the whole image are $43^{\prime} \times 38^{\prime}$

overlap, an isophote map shows no particular evidence of distortion or twisting of the isophotes of either galaxy caused by the other, other than that already referred to above for M 86 (which has been interpreted as due to star formation in the cooling hot gas or scattered starlight from dust, rather than due to interaction with $M$ 84). Of course, the very high relative velocity and radial orbit of
M 86 makes such an interaction unlikely. Other galaxies in the field also show no evidence of interaction either with M 84 and M 86 or with each other, except for NGC 4388 (see below). Caon et al. (1990) considered NGC 4387, a dwarf E5 box galaxy (Nieto \& Bender 1989), to be embedded in a common envelope of the overlapping haloes of M 84 and M 86 . However, our data suggests that the 
haloes of M 84 and M 86 may not overlap physically, and NGC 4387 shows no distortions or features that suggest it is physically interacting with either of them. Also, although NGC 4402 has an optical warped disk detected by Warmels (1988), the deep image (Fig. 2b) appears more normal. A deep, blue (IIIaJ) image including this area was produced by Kormendy \& Bahcall (1974), showing suggestions of an extended halo around these objects, although this may be due to non-uniformities in the emulsion. In our data there appears to be a brighter background in the area surrounding M 84 and M 86, hinting at a common halo, but these galaxies lie in the vignetted area of our field, near the NW corner (Fig. 6), and so we cannot be certain about the reality of this feature.

One of the most interesting galaxies of the central Virgo area is the near edge-on Seyfert 2 Sab galaxy NGC 4388 (Binggeli et al. 1985). It has a high systematic velocity with respect to the Virgo mean - its radial velocity is $2523 \mathrm{~km} \mathrm{~s}^{-1}$ (Binggeli et al. 1993) so may be a nonmember (Faber et al. 1989). However Binggeli et al. (1985) classify it as a member, while faint extensions along the major axis, principally to the west (first noted by Phillips \& Malin (1982) and visible in Fig. 2b) may be evidence of tidal interaction with other cluster members. Strong, hard X-ray emission (Hanson et al. 1990) and extended, soft X-ray emission (to a radius of $4.5 \mathrm{kpc}$, Matt et al. 1994) has been detected, although its origin is still uncertain (Antonelli et al. 1997). The dimensions of the halo in Fig. $2 \mathrm{~b}$ are $8^{\prime} \times 3^{\prime}\left(40 \times 15 \mathrm{kpc}^{2}\right)$, so the optical halo is more extensive than the $\mathrm{X}$-ray emission for this spiral galaxy.

\subsection{The giant elliptical galaxy M 89 and its "jet" and shells}

M 89, shown in Fig. 3, is an E0 elliptical galaxy with a radial velocity of $321 \pm 12 \mathrm{~km} \mathrm{~s}^{-1}$ (Binggeli et al. 1985) and is a member of the Virgo cluster. Malin (1979) reported an optical jet-like feature extending $10^{\prime}$ from the core of the galaxy, which he detected on sets of photographically amplified and co-added UKSTU IIIaJ and IIIaF plates. He also saw three shell features concentric with the galaxy. All the features were more easily seen on the blue IIIaJ plates than on the red plates. Although the nucleus of the galaxy is a compact radio source, there was no evidence of a radio feature corresponding to the jet (Heeschen 1970). However, as yet unpublished observations at $21 \mathrm{~cm}$ obtained with the VLA C array show an extended feature attached to the nucleus of the galaxy and aligned with the optical jet. Sramek (1975 and references therein) found the nucleus of M 89 to be variable at several radio frequencies, while Forman et al. (1985) found an unresolved nuclear point source with an X-ray luminosity of 3 $10^{40} \mathrm{erg} \mathrm{s}^{-1}$.

Our images of the jet-like feature and shells from the co-added array are shown in Figs. 3a-c. The normal con- trast image (Fig. 3a) shows an elliptical galaxy apparently without unusual features. Figure $3 \mathrm{~b}$ was produced by masking the original data (for more details see the last paragraph of the data reduction section of this paper). A number of M 89's features (first identified by Malin (1979) in his blue plates) are shown in Figs. 3b and 3c. The jetlike feature, the innermost shell at the SSE ( $5^{\prime}$ from the nucleus), a semicircular patch on the opposite side of the galaxy to the jet ("condensation D" of Malin (1979), only seen on his deepest IIIaJ plates) are seen in Fig. 3b, with a much greater clarity. In the much deeper Fig. 3c one can see the diffuse area at the NW (feature C of Malin (1979) which appears as a circular patch on the $R$-band images), the "jet", and an extended halo of $\sim 11.5^{\prime} \times 10.4^{\prime}$ or $\sim 58 \times 52 \mathrm{kpc}$. Note that the curved "filament" seen extending to the west of the galaxy is actually a circular defect (caused by reflection of the plate holder) on one of the original films, not quite removed by the medianfiltering addition (see Sect. 3.1).

Clark et al. (1987) obtained CCD images of the jet-like feature in $R$ and $V$ at the $1.3 \mathrm{~m}$ McGraw Hill telescope. They found the jet to be bluer than the rest of the galaxy by $V-R \approx 0.15$ and to have a total luminosity of $0.25 \%$ of that of the galaxy. They suggested that both the jet-like feature and shells were produced by a tidal encounter with a smaller galaxy. Similar interactions are well simulated by Hernquist \& Quinn (1988) and references therein.

\subsection{The interacting galaxies NGC 4438 and NGC 4435}

The apparently interacting pair NGC 4435 and NGC 4438 (radial velocities $775 \pm 15 \mathrm{~km} \mathrm{~s}^{-1}$ and $30 \pm 9 \mathrm{~km} \mathrm{~s}^{-1}$ respectively; Binggeli et al. 1985) are featured in Arp's catalogue of peculiar galaxies (Arp 1966) as number 120 . Kotanyi et al. (1983) present Einstein X-ray observations of the area of NGC 4438 showing diffuse extended emission on the west of the galaxy. Kotanyi \& Ekers (1983) also saw such emission in the $1.4 \mathrm{GHz}$ radio continuum. Both sets of authors proposed that the most likely explanation for this emission was that the interstellar gas is being swept out of NGC 4438 by a wind caused by the dense surroundings of M 87 (the projected distance between NGC 4438 and M 87 is $250 \mathrm{kpc}$ ). Kotanyi \& Ekers (1983) did not believe that there was a tidal interaction between the two galaxies because of the apparent lack of distortion of NGC 4435. However, Combes et al. (1988) present a CO map of NGC 4438 showing a central component and some molecular emission from the NW of the galaxy. Molecular gas cannot be stripped from a galaxy by intracluster medium gas, arguing in favour of a tidal interaction between the two galaxies. A numerical simulation of the interaction of the two galaxies is also presented and they argue that the galaxies's tidal interaction caused the stripping of $\mathrm{CO}$ and star formation in the molecular clouds caused the NW X-ray and radio continuum feature. Hummel \& Saikia (1991) mapped the centre of the 


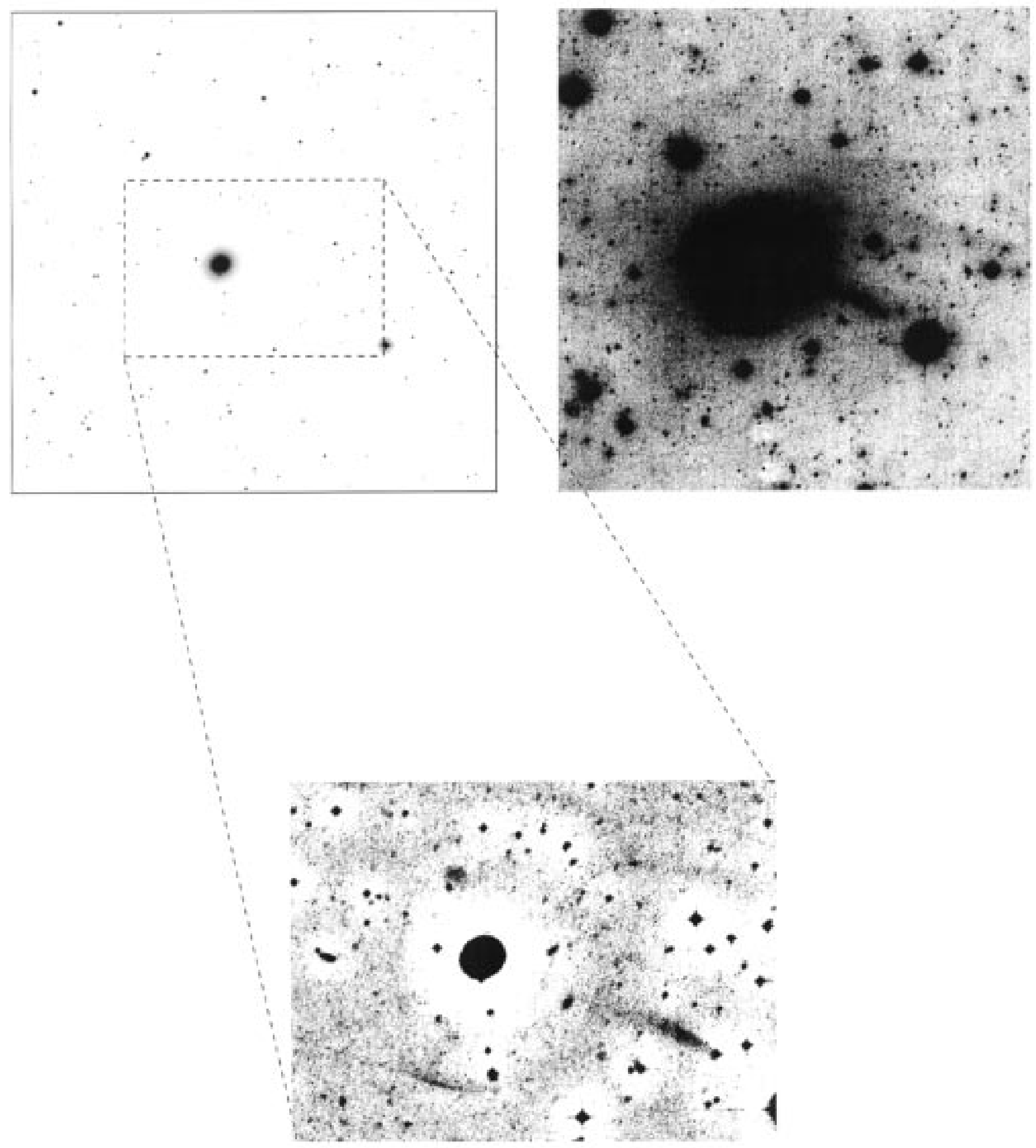

Fig. 3. a) Normal contrast image of the M 89 galaxy. The dimensions of the frame are $\sim 35^{\prime} \times 35^{\prime}$. b) High contrast presentation of the result of unsharp masking the M 89 image. The jet-like feature at the SW, the shell at SSE and the diffuse area at the NNE can be seen very clearly. The dimensions of the frame are: $\sim 18^{\prime} \times 13^{\prime}$. c) High contrast image of the M 89 galaxy covering the same field as Fig. 3a. The diffuse area at the NW (first detected by Malin 1979) and the extended halo of $\sim 11.5^{\prime} \times 10.4^{\prime}$ can be seen. The faint straight line extended NE and SW of the SE shell is very likely to be a satellite orbit 
galaxy at 1.49 and $4.86 \mathrm{GHz}$ and detected a morphologically unique (amongst the spiral galaxies) shell-like central radio source. The optical nucleus has been classified as a Liner (by Stauffer 1982; Keel 1983 and Heckman et al. 1983), but may not be the real nucleus, mostly because it does not correspond in position with the radio nucleus. There are indications that star formation in the nucleus is the most likely explanation for its strange properties. Hummel \& Saikia (1991) propose that around the nucleus, a bubble of plasma developed, expanded, and escaped by making a tunnel through the surrounding gas.

Keel \& Wehrle (1993) found two extended optical emission-line ( $\mathrm{H} \alpha$ and $[\mathrm{NII}])$ gas filaments in the vicinity of NGC 4438. They propose different origins for the two filaments and argue that only a variety of different mechanisms (interaction with the Virgo cluster's intergalactic medium, with NGC 4435 and nuclear energy release), can explain all of NGC 4438's peculiarities. Kenney et al. (1995) also detected these and other ionized filaments in $\mathrm{H} \alpha$ and $[\mathrm{NII}] \sim 1.5-3.5 \mathrm{kpc}$ from the nucleus and $\sim 5-$ $10 \mathrm{kpc}$ toward the west and southwest. They proposed that the filaments are shock-excited and as their velocities are much less than the escape velocity for the galaxy, they suggest that these filaments consist of gas disturbed by the collision and returning to the galaxy, and they delineate regions where hot gas is coming into contact with cold gas, creating layers of shocked gas at the interface. They further suggest that the features of the disturbed ISM of the system could be produced by a high-velocity ISM-ISM collision between the massive gas-rich galaxy NGC 4438 and the less massive, less gas-rich galaxy NGC 4435 (i.e. resulting from the likely tidal interaction between the two galaxies as simulated by Combes et al. 1988). NGC 4435 suffered a more severe effect from the collision. According to Kenney et al. (1995), most of its gas is captured by the much bigger companion, and they expect deeper images to show a more disturbed halo morphology for this galaxy.

Malin (1993) presented a deep image of the system of the two galaxies and detected a faint tidal tail of stars, north-northwest, pointing away from the centre (see also Kenney et al. 1995, although note that this feature is also clearly visible in the image presented by Phillips \& Malin 1982). Figure 4 presents a normal and a high contrast image of the two galaxies; the tidal tail detected by Malin (1993) can be easily seen extended up to $\sim 10^{\prime}(\sim 50$ $\mathrm{kpc}$ ) to the NNW of NGC s4435 in the very high contrast image, although there is no particular further evidence for the distortion of the halo of NGC 4435 (both galaxies however share a common halo in projection). A newlydiscovered filament of similar length can be seen extending to the SW of NGC 4438 (it is located within the curve marked in Fig. 4b). This new filament is an extension of the SW tidal tail seen in the normal contrast image. There are hints of other possible radial filaments immediately to the east of this.

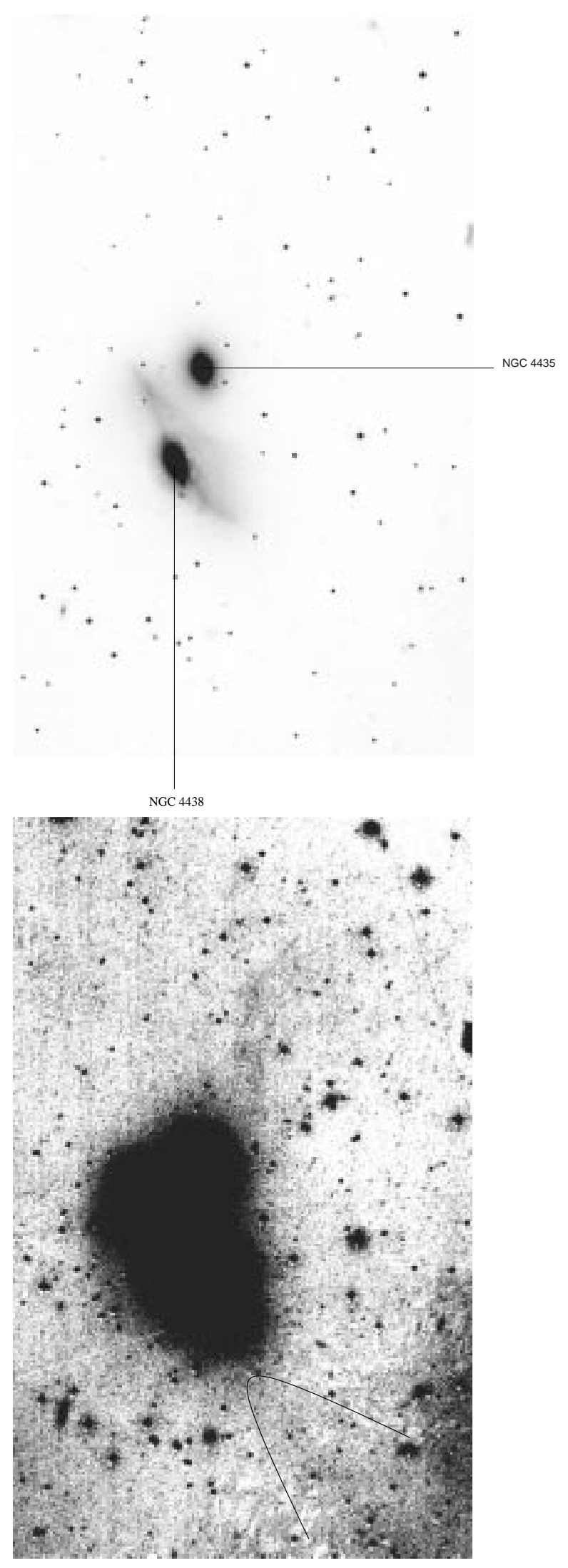

Fig. 4. High a) and very high b) contrast images of the interacting galaxies NGC 4438 and NGC 4435. The dimensions of the frame are $\sim 20^{\prime} \times 33^{\prime}$ 

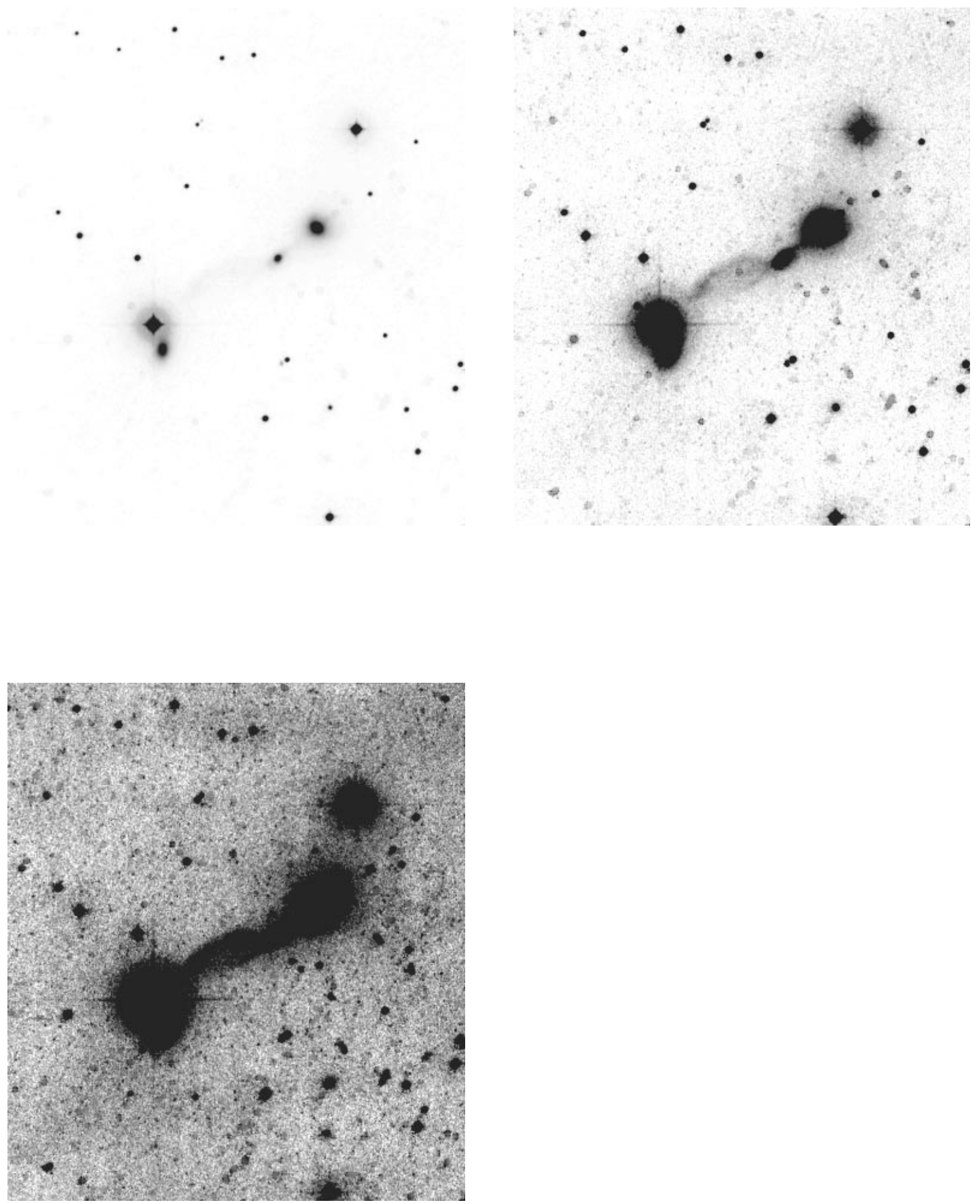

Fig. 5. Normal a), high b) and very high c) contrast image of the IC 3481, IC 3481A and IC 3483 galaxies. The details of the interaction (e.g. loop-shaped tail) can be seen on the high contrast image and the extent of the phenomenon on the very high contrast image. The dimensions of the frame are $\sim 13^{\prime} \times 14^{\prime}$ 
The expectations of Kenney et al. (1995) that NGC 4435 has the more disturbed halo are not confirmed by our deep images, although the most prominent filament (to the NW) is associated with it. These galaxies lie in a film area affected by variations in the background and many film defects not removed by the median-stacking process. Nevertheless the two faint tidal tails to NW and SW are clearly visible, there are hints of others, and it is possible that more such features could be visible in a deeper image and a more detailed study of the morphology of the halo regions could be carried out.

\subsection{The interacting group IC 3481, IC $3481 A$ \& IC 3483}

Arp (1966) was the first to mention the apparent interaction of the IC 3481, IC 3481A \& IC 3483 galaxies. This interaction is displayed in Fig. 5, which contains three images at different contrast, which display a number of remarkable newly-detected faint features. The normal contrast image, Fig. 5a, is similar to the one presented by Arp (1966), although much more extended; the interaction between IC 3481 \& IC $3481 \mathrm{~A}$ is visible, but the tail towards IC 3483 is very faint. On the higher contrast image, Fig. 5b, the tail of the interaction and its properties are clearly seen: a loop-like structure is formed with one apex apparently just touching IC 3481A, and the other apex apparently close to IC 3483 . On the very high contrast image, Fig. 5c, one can see the extent of the interaction around the IC $3481 \mathrm{~A}$ galaxy and the common halo surrounding IC 3481 and IC $3481 \mathrm{~A}$. The details of how the loop-like structure connects (or not) to IC3483 is hidden by the halo of the bright foreground star. $R$ magnitudes of $12.9,15.9$ can be measured from the CCD data obtained from the JKT, for the galaxies IC 3481 and IC 3481A respectively. Only a lower limit of $16.5 \mathrm{mag}$ for the newlydetected faint features can be reported as the frame of the CCD data was not extensive enough.

Although visual inspection is strongly suggestive that all three galaxies are interacting and connected by newly discovered faint filamentary material, there is a strong possibility that IC 3483 is not involved in this interaction, indicated by its H I radial velocity: $128 \pm 10 \mathrm{~km} \mathrm{~s}^{-1}$, (Binggeli et al. 1993), while IC 3481 \& IC 3481A have radial velocities of $7086 \pm 56 \mathrm{~km} \mathrm{~s}^{-1}$ (Binggeli et al. 1985) \& $7304 \pm 56 \mathrm{~km} \mathrm{~s}^{-1}$ (de Vaucouleurs et al. 1991) respectively. IC 3481 and IC3481A are therefore objects in the background to the Virgo cluster, and the interaction is likely to involve only these two galaxies.

\subsection{Variation of background photographic density}

A large-scale variation in background photographic density is apparent in a high contrast image of the co-added array (see Fig. 6). Inspection of scans of individual films

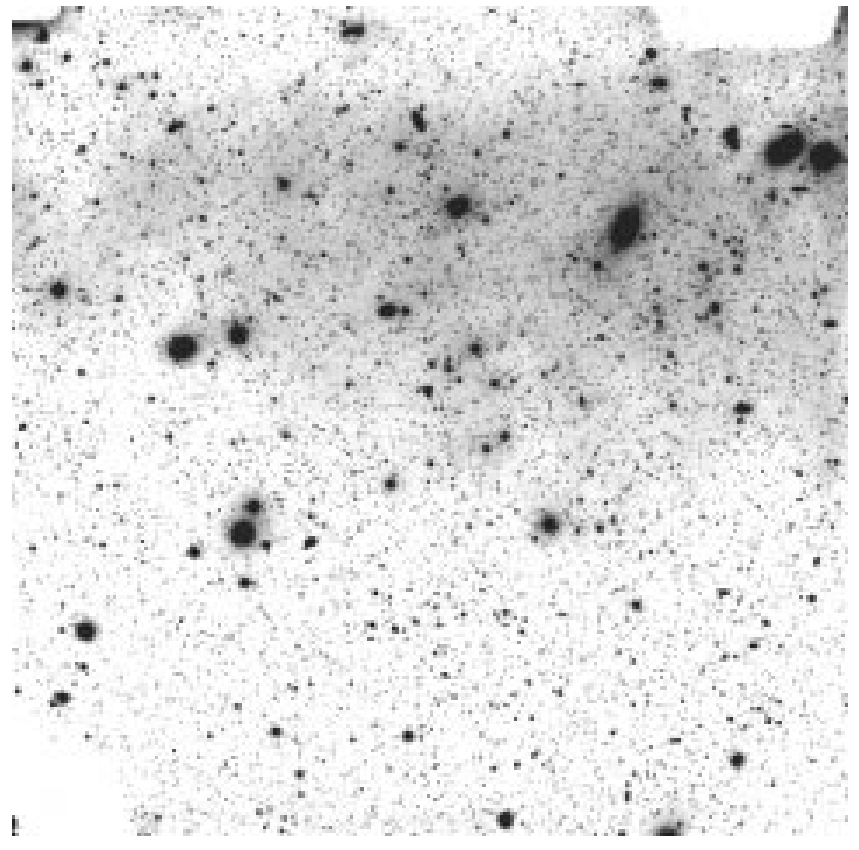

Fig. 6. Very high contrast image of the whole $\left(\approx 6.2^{\circ} \times 6.2^{\circ}\right)$ field covered by the Schmidt films. A large scale, marginal, variation in photographic density is apparent. This may be a real detection of the luminous, inter-galactic, medium, or simply an artefact as consequence of the variation in sensitivity over this large field

reveals that the morphology of this background variation changes considerably from film to film, suggesting that, for individual films at least, that it is dominated by variations in emulsion sensitivity across the film.

We further investigated this large scale variation using three IIIaF plates of the north-east area of the Virgo cluster also taken with the UKST. This second field overlaps with our Virgo SE field. The OG590 filter (described above) was used for the IIIaF plates to give results close to the standard $R$-band (UKSTU handbook). We co-added the scans of three IIIaF Virgo NE plates using exactly the same procedure, as discussed in Sect. 2.

The morphology of the possible background emission in Fig. 6 is such that the brightest regions are seen in the central parts of the cluster towards the north and northwestern parts of the field. As this is much what we would have anticipated, it appears that we could be detecting genuine optical, intra-cluster, medium emission, in which case the co-addition with median-filtering of the thirteen film scans would not be dominated by the variations in sensitivity across individual films. However, the reality of this most extensive emission must remain uncertain until further observations are made. 


\section{Conclusion}

This paper illustrates preliminary results obtained from the digital co-addition of 13 APM scans of Kodak TechPan UKST films of a field containing the SE part of the Virgo cluster. With this technique we can detect previously unseen low surface brightness features of galaxies over the whole of the $\approx 6.2^{\circ} \times 6.2^{\circ}$ area scanned, producing data with an increased dynamic range and which can be calibrated photometrically using CCD observations of objects within the field. Hence we can make full quantitative use of this data array, and this important calibration will be reported in a future paper (some preliminary calibration results are given in this paper). Also with such digital data, processing techniques such as removal of stellar images can be performed, while methods such as "unsharp masking" are greatly facilitated. Mosaicing of CCD frames is not yet competitive in terms of covering such large fields in equivalent observing and processing times, and has problems with matching backgrounds at the edges of frames. Many of the galaxies reported here would require mosaicing of large numbers of contiguous CCD fields to cover their apparent area.

While processing the film scans we noted that there were variations in emulsion sensitivity over the wide field of the films, varying in position from film to film. In the co-added image it appears that the faintest structures we detect are reliable up to scales of about 1 square degree on average. In Fig. 6 we see a strong suggestion of very faint intra-cluster medium emission occupying the northern part of the field (central area of the cluster), but the reality of these structures needs to be tested with accurate calibration of each film, further films and independent measurements. This is one of our priorities for future work, as is investigating the causes of the apparent variation in emulsion sensitivity across each film.

Nevertheless the TechPan films clearly provide good imaging quality, even with the slight blurring introduced by digital aligning and co-addition (errors in alignment are only a small fraction of a pixel), while new faint features of galaxies have been discovered, even though many of these galaxies have been studied before (some previous studies have been at blue wavebands, where the relatively faint sky level makes low surface brightness structures easier to detect). When the data array is satisfactorily calibrated, we will present the accurate surface brightnesses for the features detected here and the gain in magnitude obtained by the co-addition.

With a single film features should be detected at surface brightnesses as low as $27 R$ mag $\operatorname{arcsec}^{-2}$ (Phillipps \& Parker 1993). As the co-addition of 13 films should reduce the sky photon noise per pixel by a factor $\sqrt{\frac{2 \times 13}{\pi}}(\approx 2.9)$, then features as low as $28.1 R$ mag $\operatorname{arcsec}^{-2}$ should be visible in the co-added array. The CCD data from the sky area around the galaxies IC 3481 \& IC 3481A were used to obtain a preliminary photometric calibration and a sky noise level of $27.9 \pm 0.3 \mathrm{mag} \operatorname{arcsec}^{-2}$ was measured for the co-added array for the area around these galaxies.

The problems associated with the processing of large (222 Mb) data files have been overcome by a combination of modifying existing Starlink software, and writing new software. These software items have been used to provide new ways of implementing techniques such as alignment, normalisation, co-addition, correction for vignetting effects, and removal of stellar features. Positional information was also stored in the final co-added array.

Some new astrophysical results are presented here. Filaments are seen associated with the apparently interacting galaxies NGC 4435 and NGC 4438. Connecting filaments are seen between the galaxies IC 3481 and IC 3481A for the first time, and a further "loop" of filaments apparently connecting these two galaxies with IC 3483 (though we note the discrepant redshift of the latter). Also more high-quality images are presented of previously detected features such as the N filament associated with NGC 4435 and NGC 4438, and the shells and "jet" features of M 89. Also deep images are presented of the giant haloes surrounding the ellipticals M 87, M 84 and M 86, although, perhaps surprisingly, no trace of distortions are found in the halo shapes of galaxies apparently adjacent to them, some of which might have been thought to be interacting with them. Co-addition of further films will clarify various uncertain features (e.g. whether or not there are any more shells in the vicinity of M 89 or further filaments associated with NGC 4435/8).

Complete CCD calibration and multicolour photometry will be important for the scientific interpretation of these features. We will also estimate the mass of luminous material contained within the haloes of galaxies in the field. Comparison of the revealed halo sizes with the extent of X-ray emission detected by ROSAT and other $\mathrm{X}$-ray satellites show that there is no consistent correlation between the $R$-band and X-ray haloes. The X-ray haloes can be much larger than the optical haloes (as in the case of M 87) or much smaller, nevertheless the combination of visible ("red-emitting") mass in the haloes detected here and the X-ray-emitting mass can provide new estimates of the total luminous baryonic masses of galaxies.

Acknowledgements. We are particularly grateful to the staff of both the UKSTU and the ROE for the provision of the photographic material, their help on various occasions and the useful discussions. We also thank the Royal Greenwich Observatory (RGO) and specially Dr. M. Irwin for the scanning of the films. Many thanks also to Dr. B. Kellett for his hospitality and the interesting discussions, and Dr. R.J.R. Williams, Dr. Q.A. Parker, Dr. S. Phillipps and J.-M. Schwartzenberg for further interesting and useful discussions. 


\section{References}

Arp H., 1966, ApJS 14, 1

Arp H., Bertola F., 1969, ApJ 4, L23

Antonelli L.A., Matt G., Piro L., 1997, A\&A 317, 686

Binggeli B., Popescu C.C., Tammann G.A., 1993, A\&AS 98, 275

Binggeli B., Sandage A., Tammann G.A., 1985, AJ 90, 1681

Caon N., Capacciolli M., Rampazzo R., 1990, A\&AS 86, 429

Clark G.W., Plucinsky P., Ricker G., 1987, in IAU Symposium 127, "Structure and Dynamics of Elliptical Galaxies", de Zeeuw T. (ed.). Reidel, Dordrecht, Holland, p. 457

Combes F., Dupraz C., Casoli F., Pagani L., 1988, A\&A 203, L9

Fabbiano G., Kim D.-W., Trinchieri G., 1992, ApJS 80, 531

Faber S.M., Wenger G., Burstein D., et al., 1989, ApJS 69, 763

Fabian A.C., Schwarz J., Forman W., 1980, MNRAS 192, 135

Fabricant D., Gorenstein P., 1983, ApJ 267, 535

Forman W., Jones C., Tucker W., 1985, ApJ 293, 102

Hanson C.G., Skinner G.K., Eyles C.J., Willmore A.P., 1990, MNRAS 242, 262

Heeschen D.S., 1970, AJ 75, 523

Heckman T.M., Balick B., Van Breugel W.J.W., Miley G.K., 1983, ApJ 265L, 1

Hernquist L., Quinn P.J., 1988, ApJ 331, 682

Hummel E., Saikia D.J., 1991, A\&A 249, 43

Jacoby G.H., Ciardullo R., Ford H.C., 1990, ApJ 356, 332

Keel W.C., 1983, ApJ 269, 466

Keel W.C., Wehrle A.E., 1993, AJ 106, 236

Kemp S.N., A\&A 282, 425

Kemp S.N., Meaburn J., 1991a, MNRAS 251, 10

Kemp S.N., Meaburn J., 1991b, MNRAS 252, 27

Kemp S.N., Meaburn J., 1993, A\&A 274, 19

Kemp S.N., Meaburn J., 1994, A\&A 289, 39

Kemp S.N., Meaburn J., 1995, in ASP Conf. Ser. 84, "The Future Utilisation of Schmidt Telescopes", Chapman J., Cannon R., Harrison S., Hidayat B. (eds.). San Francisco, CA, p. 200

Kenney J.D.P., Rubin V.C., Planesas P., Young J.S., ApJ 438, 135
Kodak Publication P-255, 1981, "KODAK Technical Pan Film"

Kormendy J., Bahcall J.N., 1974, AJ 79, 671

Kotanyi C.G., Ekers R.D., 1983, A\&A 122, 267

Kotanyi C.G., van Gorkom J.H., Ekers R.D., 1983, ApJ 273, L7

Laing R.A., Riley J.M., Longair M.S., MNRAS 204, 151

Laing R.A., Bridle A.H., 1987, MNRAS 228, 577

Malin D.F., 1979, Nat 277, 279

Malin D.F., 1981, Am. Ast. Photo-Bull., No. 27, 4

Malin D.F., 1993, A View of the Universe. Cambridge Univ. Press

Matt G., Piro L., Antonelli L.A., Fink H.H., Meurs E.J.A., Perola G.C., 1994, A\&A 292, L13

Mould J., Aaronson M., Huchra J., 1980, ApJ 238, 458

Nieto J.-L., Bender R., 1989, A\&A 215, 266

Nulsen P.E.J., Böhringer H., 1995, MNRAS 274, 1093

Nulsen P.E.J., Carter D., 1987, MNRAS 225, 939

Phillips M.M., Malin D.F, 1982, MNRAS 199, 905

Phillipps S., Parker Q.A., 1993, MNRAS 265, 385

Prugniel P., Nieto J.-L., Simien F., 1987, A\&A 173, 49

Rangarajan F.V.N., White D. A., Ebeling H., Fabian A.C., 1995, MNRAS 277, 1047

Rood, H.J., 1965, AJ 70, 689

Russell K.S., Malin D.F., Savage A., Hartley M., Parker Q.A., 1992, "The use of Eastman Kodak 4415 film in the UKST", in "Digitised Optical Sky Surveys", MacGillivray H.T. and Thomson E.B. (eds.). Kluwer Academic Publishers, Dordrecht, p. 23

Sramek R.A., 1975, ApJ 198, L13

Stauffer J.R., 1982, ApJ 262, 66

UKSTU Handbook, ROE, 1983

Vaucouleurs G. de, Vaucouleurs A. de, Corwin H.G., Jr., et al., 1991, Third Reference Catalogue of Bright Galaxies. Springer-Verlag, New York

Warmels R.H., 1988, A\&AS 72, 57

Weil M.L., Bland-Hawthorn J., Malin D.F., 1997, ApJ 490, 664

White D.A., Fabian A.C., Forman W., Jones C., Stern C., 1991, ApJ 375, 35 